\title{
Impact of Model Detail of Synchronous Machines on Real-time Transient Stability Assessment
}

Weckesser, Johannes Tilman Gabriel; Jóhannsson, Hjörtur; Østergaard, Jacob

Published in:

Proceedings of 2013 IREP Symposium

Link to article, DOI:

10.1109/IREP.2013.6629364

Publication date:

2013

Document Version

Publisher's PDF, also known as Version of record

Link back to DTU Orbit

Citation (APA):

Weckesser, J. T. G., Jóhannsson, H., \& Østergaard, J. (2013). Impact of Model Detail of Synchronous Machines on Real-time Transient Stability Assessment. In Proceedings of 2013 IREP Symposium IEEE.

https://doi.org/10.1109/IREP.2013.6629364

\section{General rights}

Copyright and moral rights for the publications made accessible in the public portal are retained by the authors and/or other copyright owners and it is a condition of accessing publications that users recognise and abide by the legal requirements associated with these rights.

- Users may download and print one copy of any publication from the public portal for the purpose of private study or research.

- You may not further distribute the material or use it for any profit-making activity or commercial gain

- You may freely distribute the URL identifying the publication in the public portal 


\title{
Impact of Model Detail of Synchronous Machines on Real-time Transient Stability Assessment
}

\author{
Tilman Weckesser, Hjörtur Jóhannsson and Jacob Østergaard \\ Centre for Electric Power and Energy, Department \\ of Electrical Engineering, Technical University of Denmark, 2800 Lyngby
}

\begin{abstract}
In this paper, it is investigated how detailed the model of a synchronous machine needs to be in order to assess transient stability using a Single Machine Equivalent (SIME). The results will show how the stability mechanism and the stability assessment are affected by the model detail. In order to identify the transient stability mechanism, a simulation with a high-order model was used as reference. The Western System Coordinating Council System (WSCC) and the New England \& New York system are considered and simulations of an unstable and a stable scenario are carried out, where the detail of the machine models is varied. Analyses of the results suggest that a $4^{\text {th }}$-order model may be sufficient to represent synchronous machines in transient stability studies.
\end{abstract}

\section{Introduction}

Today's society is highly dependent on a stable and secure supply of electric power. In the future, this is not expected to change. The shift from fossil energy sources to renewable energy sources, which can be observed in many countries around the world and the aim of reaching a Danish society with minimal dependency on fossil fuels [1], represents a great challenge for the power system. These ambitious plans can only be achieved, when a large share of the electric power generation uses renewable energy sources, whose energy sources are noncontrollable sources such as wind and solar radiation [1]. The integration of such non-controllable sources will lead to increased fluctuations of the power system's operating point. This will make the planning of secure and stable operation hours ahead no longer feasible and the need for real-time stability assessment tools will arise.

In [2], [3], [4] phasor measurement technology is said to be the technology enabling the development of real-time wide area monitoring and control applications [5], [6].

Consequently, recently developed stability assessment methods try to solve the task of on-line security and stability assessment by utilizing synchronized phasor measurements. This is done by either developing an

This research was supported by the Danish Strategic Research Council. entirely new approach such as in [7], [8] or by adaption of an off-line method to real-time operation, e.g. in [9], [10]. In [11] the adaptability of various direct transient stability assessment (TSA) methods to real-time operation was investigated. The analysis showed that the SIME method allows the fastest TSA. The method utilizes input from time-domain simulation. In order to achieve fast computation, it is desirable to reduce the number of differential equations to be solved. Hence, in the following it is investigated, how detailed the model of a synchronous generator needs to be to depict the transient stability mechanism accurately enough to allow a detection of the instability and its origin. However, it should be noted that in this work the focus is solely on assessing first swing instability.

In this work the RAMSES software [12] developed at the University of Liège was used to perform the dynamic time-domain simulations.

\section{Transient Stability Assessment}

Transient stability assessment (TSA) analyzes the system's ability to sustain large transient disturbances such as loss of generation or failure on transmission facilities [13]. These disturbances lead to large excursion of the machines rotor angle, which are described by the strongly non-linear relations governing the dynamics in power systems. Consequently, transient stability cannot be assessed through linearization of the system equations.

\section{Direct methods}

In order to allow fast transient stability assessment, direct TSA methods were developed. These methods try to avoid explicitly solving the system differential equations. One of the main approaches is based on Lyapunov's method [14] and a second main approach is applying the equal area criterion [15].

Lyaponov's method. In order to apply Lyapunov's method the system is described by a transient energy function, which allows determining transient stability after identification of the stable and unstable equilibrium point (UEP) of the post-fault system [14]. However, since the system is described by one single transient energy 
function, it is difficult to determine the origin of the instability. One of the most recent developments applying this approach is the so called BCU method [16].

Equal Area Criterion. The equal area criterion (EAC) allows assessing transient stability of one-machine infinite bus (OMIB) systems without explicitly solving the swing equation [13]. The criterion essentially states that the system needs to be capable of absorbing the kinetic energy of the generator gained during the fault. The gained kinetic energy and the energy absorbing capability are represented by areas defined by the mechanical power and the $P-\delta$-curve, which describes the non-linear relation of the electric power injection and the rotor angle. Hence, with certain assumptions and simplifications the stability criteria can be formulated as an integral.

This approach was further developed with the extended equal area criterion [17] and most recently with the hybrid method SIME [15].

An analysis of the computational burden of the two mentioned methods with respect to real-time implementation was carried out in [11]. The results suggest that methods based on the EAC are faster than methods using Lyapunov's method. Hence, in the following SIME is considered for the fast screening method.

\section{Single Machine Equivalent (SIME) Method}

Transient stability assessment with a SIngle Machine Equivalent (SIME) is based on the Equal Area Criterion and considered to be a hybrid method, since it combines the advantages of a direct method and time-domain simulation. A detailed description of the method can be found in [15].

Concept. The SIME method determines in each simulation step parameters of a candidate one-machine infinite bus (OMIB) system, which describes the dynamics of a critical generator group and a group of remaining (non-critical) generators. The machines in the system are split up into the critical and the non-critical group corresponding to their rotor angles. The machines in each group are aggregated and the parameters of the OMIB system are determined. After aggregation of the machines the transient stability of the resulting OMIB can be assessed using the equal area criterion.

Transient stability margin. The stability margin of such a system can be calculated as follows [15].

$$
\eta=-\int_{\delta_{i}}^{\delta_{u}} P_{a} d \delta-1 / 2 M \omega_{i}^{2}
$$

$M \quad$ Inertia coefficient of candidate OMIB $\left[s^{2} / \mathrm{rad}\right]$

$P_{a} \quad$ Accelerating power of candidate OMIB [pu]

$\delta_{i} \quad$ Current rotor angle of candidate OMIB [ $\left.\mathrm{rad}\right]$

$\delta_{u} \quad$ Rotor angle at UEP of candidate OMIB [ $\left.\mathrm{rad}\right]$

$\eta \quad$ Transient stability margin in [ $\mathrm{rad}]$

$\omega_{i} \quad$ Current rotor speed of candidate OMIB $[\mathrm{rad} / \mathrm{s}]$
Here a negative margin represents an unstable case and a positive margin a stable case [15]. The unit of the stability margin computed with eq. (7) is radians. Often the margin is normalized with respect to the inertia coefficient of the OMIB, which results in a normalized stability margin with unit $(\mathrm{rad} / \mathrm{s})^{2}$.

Implementation. In this case the implementation of SIME is used to carry out a fast screening of the current system condition with respect to $\mathrm{N}-1$-contingencies and first swing transient stability. The implementation is derived from E-SIME, e.g. described in [15], [18]. E-SIME uses measurements of the post-fault system, which are acquired in real-time. These measurements are replaced in this implementation by data received from the timedomain simulation software RAMSES. This implementation of SIME is called preventive SIME [15]. With the application of SIME the aim is to get an early stop criterion for the simulation, which declares a contingency to be definitely stable or unstable.

In order to determine first swing stability of the particular contingency scenario, a time-domain simulation is run until at least three data sets of the post-fault system are available. Then the predictive SIME method is executed the first time and, subsequently, the stability assessment is updated with data sets acquired in each successive simulation step.

In the following the procedure of preventive SIME is described in more detail (see also [11] and [15]).

Step 1: Consider the first three data sets received from the time-domain simulation in the post-fault configuration. Step 2: Predict the rotor angle of each individual generator some time ahead $(\sim 100 \mathrm{~ms})$ using Taylor series expansion truncated after the quadratic term. Step 3: Rank generators according to the predicted rotor angles and identify the critical machine candidates looking for the maximum angular deviation of two successive machines. The machines above this gap form the candidate critical machines and the ones below the candidate the noncritical machines. The machines are then aggregated accordingly and the candidate OMIB is determined. Step 4: Subsequently, the parameter of the candidate OMIB can be computed and, utilizing the accelerating power and the rotor angle from at least three successive data sets, the $P_{a}-\delta$ curve can be estimated as follows.

$$
P_{a}(\delta)=a \delta^{2}+b \delta+c
$$

Note: First, the parameters $a, b$ and $c$ can be determined using the three acquired data sets and, in the following, with additional acquired data the prediction can be refined using a weighted least square technique. Step 5: The angle $\delta_{u}$ at the unstable equilibrium point is determined by solving $P_{a}\left(\delta_{u}\right)=0$ and by checking if the instability conditions of eq. (3) are met.

$$
P_{a}\left(\delta_{u}\right)=0 ; \quad \dot{P}_{a}\left(\delta_{u}\right)>0
$$

If the instability conditions (3) are not met, then a new set of data is acquired and the procedure is repeated from Step 2. If the conditions are met the stability margin is computed utilizing eq. (1), then a new set of data is 
acquired and the Steps 2 to 5 are repeated to refine the computed $\delta_{u}$ and the estimated stability margin $\eta$. The procedure is terminated when the stability margin converged to a constant value or the angle of maximum excursion $\delta_{r}$ is reached where the following conditions are met.

$$
P_{a}\left(\delta_{r}\right)<0 ; \omega_{r}=0
$$

Since the method to determine transient stability requires time-domain simulation a speed up of the assessment can be achieved, when the model detail of the power system components, used in the simulation, can be reduced. Hence, in the following the method is tested with reduced order synchronous machine models.

\section{Synchronous machine models}

In the following section the described synchronous machine (SM) models were adopted from [19]. The $6^{\text {th }}$ and $4^{\text {th }}$-order model are readily integrated in RAMSES. The $3^{\text {rd }}$-order and $2^{\text {nd }}$-order model were realized through appropriate selection of the time constants in the $4^{\text {th }}$-order model.

\section{Four winding model ( $6^{\text {th }}$-order $)$}

In the $6^{\text {th }}$-order model, four windings are considered, two on the q-axis and two on the d-axis. However, the network and stator transients are neglected. According to [13] the dynamics introduced by these transients may be neglected and this will lead to slightly conservative results, which is preferable in stability studies and in particular for fast screening where all critical and unstable scenarios should be identified.

In dynamic analysis, when using the $6^{\text {th }}$-order model, the synchronous machine is described by the following six equations [19].

$$
\begin{aligned}
& T_{d o}^{\prime} \frac{d E_{q}^{\prime}}{d t}=-E_{q}^{\prime}-\left(X_{d}-X_{d}^{\prime}\right)\left[I_{d}\right. \\
&-\frac{X_{d}^{\prime}-X_{d}^{\prime \prime}}{\left(X_{d}^{\prime}-X_{l s}\right)^{2}}\left(\psi_{1 d}\right. \\
&\left.\left.+\left(X_{d}^{\prime}-X_{l s}\right) I_{d}+E_{q}^{\prime}\right)\right] \\
&+E_{f d} \\
& T_{d o}^{\prime \prime} \frac{d \psi_{1 d}}{d t}=- \psi_{1 d}+E_{q}^{\prime}-\left(X_{d}^{\prime}-X_{l s}\right) I_{d} \\
& T_{q o}^{\prime} \frac{d E_{d}^{\prime}}{d t}=-E_{d}^{\prime}+\left(X_{q}-X_{q}^{\prime}\right)\left[I_{q}\right. \\
&-\frac{X_{q}^{\prime}-X_{q}^{\prime \prime}}{\left(X_{q}^{\prime}-X_{l s}\right)^{2}}\left(\psi_{2 q}\right. \\
&\left.\left.+\left(X_{q}^{\prime}-X_{l s}\right) I_{q}+E_{d}^{\prime}\right)\right] \\
& T_{q o}^{\prime \prime} \frac{d \psi_{2 q}}{d t}=- \psi_{2 q}+E_{d}^{\prime}-\left(X_{q}^{\prime}-X_{l s}\right) I_{q} \\
& \frac{d \delta}{d t}=\omega_{s}-\omega_{s}
\end{aligned}
$$

$E_{d, q}^{\prime}$

$E_{f d}$

H

$I_{d, q}$

$T_{d o, q o}^{\prime}$

$T_{d o, q o}^{\prime \prime}$

$T_{F W}$

$T_{M}$

$X_{d, q}^{\prime}$

$X_{d, q}^{\prime \prime}$

$X_{l s}$

$\delta$

$\psi_{1 d}$

$\psi_{2 q}$

$\omega$

$\omega_{s}$

Equation (5) and (6) describe the dynamics in the d-axis, while equation (7) and (8) describe the dynamics in the qaxis. Equation (9) and (10) represents the well-known swing equation.

In all models additional damping may be added through the optional torque component $T_{F W}$, which introduces a damping torque proportional to the rotational speed.

\section{Two-axis model $\left(4^{\text {th }}\right.$-order $)$}

In the $4^{\text {th }}$-order two axis model, the damper winding dynamics $\psi_{1 d}$ and $\psi_{2 q}$ are neglected. As described in [19], this is achieved by setting $T_{d o}^{\prime \prime}$ and $T_{q o}^{\prime \prime}$ equal to zero, which leads to the following mathematical description of the synchronous machine.

$$
\begin{gathered}
T_{d o}^{\prime} \frac{d E_{q}^{\prime}}{d t}=-E_{q}^{\prime}-\left(X_{d}-X_{d}^{\prime}\right) I_{d}+E_{f d} \\
T_{q o}^{\prime} \frac{d E_{d}^{\prime}}{d t}=-E_{d}^{\prime}+\left(X_{q}-X_{q}^{\prime}\right) I_{q} \\
\frac{d \delta}{d t}=\omega-\omega_{s} \\
\frac{2 H}{\omega_{s}} \frac{d \omega}{d t}=T_{M}-E_{q}^{\prime} I_{q}-E_{d}^{\prime} I_{d}-\left(X_{q}^{\prime}-X_{d}^{\prime}\right) I_{d} I_{q} \\
-T_{F W}
\end{gathered}
$$

It should be noted that, while the $\psi_{1 d}$ and $\psi_{2 q}$ dynamics are neglected, the $E_{d}^{\prime}$ and $E_{q}^{\prime}$ dynamics are maintained. 
Table 1 Parameter of the three generators in the WSCC test system on machine basis adopted from [15]

In the one-axis model, the representation of the synchronous machine is reduced by another degree. Therefore, the damper windings dynamics $E_{d}^{\prime}$ are eliminated. This reduction is achieved by setting $T_{q o}^{\prime}$ equal to zero and results in the following equations [19].

$$
\begin{gathered}
T_{d o}^{\prime} \frac{d E_{q}^{\prime}}{d t}=-E_{q}^{\prime}-\left(X_{d}-X_{d}^{\prime}\right) I_{d}+E_{f d} \\
\frac{d \delta}{d t}=\omega-\omega_{s} \\
\frac{2 H}{\omega_{s}} \frac{d \omega}{d t}=T_{M}-E_{q}^{\prime} I_{q}-\left(X_{q}-X_{d}^{\prime}\right) I_{d} I_{q} \\
-T_{F W}
\end{gathered}
$$

\section{Classical model ( $2^{\text {nd }}$-order)}

The last synchronous machine model, which is considered in this analysis, is the classical model. In this model the voltage behind the transient reactance is assumed to be constant. For such a representation only the swing equation is needed to describe the dynamics of a synchronous machine.

$$
\begin{gathered}
\frac{d \delta}{d t}=\omega-\omega_{s} \\
\frac{2 H}{\omega_{s}} \frac{d \omega}{d t}=T_{M}-\frac{E^{\prime} V_{T}}{X_{d}^{\prime}} \sin \left(\delta-\theta_{T}\right)-T_{F W}
\end{gathered}
$$

$E^{\prime} \quad$ Internal voltage behind transient reactance

$V_{T} \quad$ Terminal voltage

\section{Test systems and scenarios}

Two test systems, namely the Western System Coordinating Council system as well as the New England \& New York system, were considered for the analysis. For each of the test systems a transient stable and a transient unstable test scenario were considered.

\section{Western System Coordinating Council (WSCC)}

The WSCC system is a 9-bus system with 3 generators. The load flow and the dynamic data were adopted from [19]. However, in order to further stress the system, the system loading was increased by approximately $50 \%$. The power generation of the generators was increased correspondingly.

The parameters of the synchronous machine models can be seen in Table 1 . In the original WSCC system the generators are solely described by a $4^{\text {th }}$-order model. Consequently, for the $6^{\text {th }}$-order model standard parameter were added, which were taken from [13].

In order to allow assessment of solely the influence of the generator model, the excitation systems were removed from the model and the excitation was assumed to be manually. Furthermore, no governor model was implemented.

\begin{tabular}{|c|c|c|c|}
\hline Parameter & GEN-1 & GEN-2 & GEN-3 \\
\hline \hline$M V A_{\text {base }}$ & 500.0 & 300.0 & 300.0 \\
\hline$H[s]$ & 4.728 & 2.133 & 1.003 \\
\hline $\mathrm{X}_{d}[\mathrm{pu}]$ & 0.7300 & 2.6874 & 3.9375 \\
\hline$X_{d}^{\prime}[\mathrm{pu}]$ & 0.3040 & 0.3594 & 0.5439 \\
\hline$X_{d}^{\prime \prime}[\mathrm{pu}]^{1}$ & 0.2500 & 0.2500 & 0.3000 \\
\hline $\mathrm{X}_{q}[\mathrm{pu}]$ & 0.4845 & 2.5935 & 3.7734 \\
\hline$X_{q}^{\prime}[\mathrm{pu}]$ & 0.3040 & 0.3594 & 0.5439 \\
\hline$X_{q}^{\prime \prime}[\mathrm{pu}]^{1}$ & 0.2500 & 0.2500 & 0.3000 \\
\hline$T_{d o}^{\prime}[\mathrm{s}]$ & 8.690 & 6.000 & 5.890 \\
\hline$T_{d o}^{\prime \prime}[s]^{1}$ & 0.030 & 0.045 & 0.030 \\
\hline$T_{q o}^{\prime}[s]$ & 0.310 & 0.535 & 0.600 \\
\hline$T_{q o}^{\prime \prime}[\mathrm{s}]^{1}$ & 0.040 & 0.035 & 0.040 \\
\hline
\end{tabular}

${ }^{1}:$ Standard parameter chosen from [13]

In order to assess how detailed the synchronous machine model needs to be to depict the instability mechanism of the first swing accurately and to allow correct stability assessment using SIME, two test scenarios are considered, an unstable and a stable case.

In [20] it is stated that it is sufficient to represent turbogenerators by their $6^{\text {th }}$-order model in stability analysis. Therefore, the simulations using the $6^{\text {th }}$-order generator model serve as a reference case and it is assumed to reflect reality sufficiently.

Unstable case. In the unstable case, the stressed system is driven to instability through the loss of the heavily loaded line connecting bus 7 and $8100 \mathrm{~ms}$ after simulation begin. In this case, it is assumed that the line is tripped due to overloading and it is not reconnected.

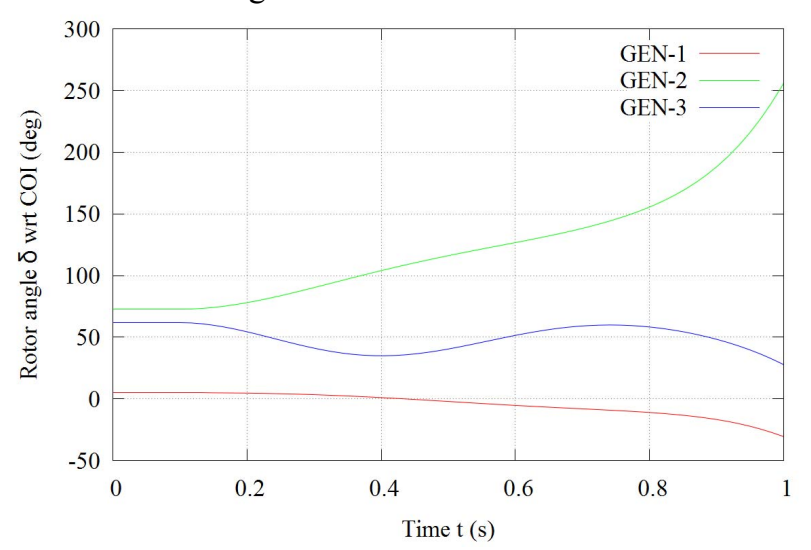

Fig. 1: Unstable response of the rotor angles of the generators to the transient disturbance

Figure 1 shows that the loss of the heavily loaded transmission line triggers a first swing instability and causes generator 2 to lose synchronism.

Stable case. In the stable case the same fault as in the unstable case is considered, but the transmission line is reconnected after $150 \mathrm{~ms}$. This reconnection leads to a relative deceleration of the affected generators and 
eventually the system reaches a new steady state operating point. Figure 2 displays the rotor angle response of all three generators.

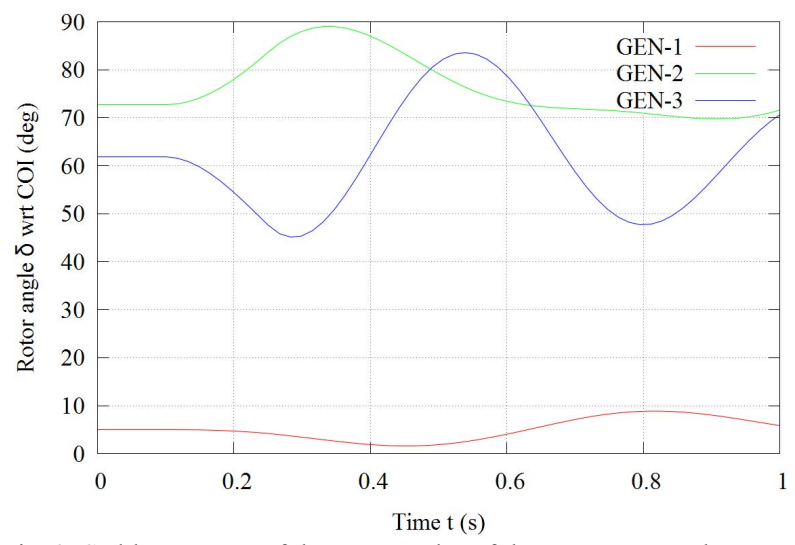

Fig. 2: Stable response of the rotor angles of the generators to the transient disturbance

\section{New England and New York}

The second test system represents the New England and New York system. The system was adopted from Graham Rogers [21] and consists of sixteen generators and 68 buses. The synchronous generators are modeled using a $6^{\text {th }}$-order model, thermal turbine/governor model and static exciters. All generators but generator 7 and 14 are equipped with a PSS. The parameters and a detailed model description can be found in [21]. In the following two transient stability scenarios are considered.

Stable case. The stable case is adopted from chapter 5 in [21]. The considered fault is a three phase short circuit very close to bus 21 applied $1 s$ after simulation begin. The fault is cleared after $150 \mathrm{~ms}$ by opening the breakers at both ends of the transmission line connecting bus 16 and bus 21 . The case represents a marginal stable scenario, which is apparent due to the large angular excursion of the rotor angles of the generators 6 and 7 . Figure 3 shows the rotor angles over time, when the synchronous machines are represented by a $6^{\text {th }}$-order model. Like before this simulation will serve as reference for the TSA when using SIME.

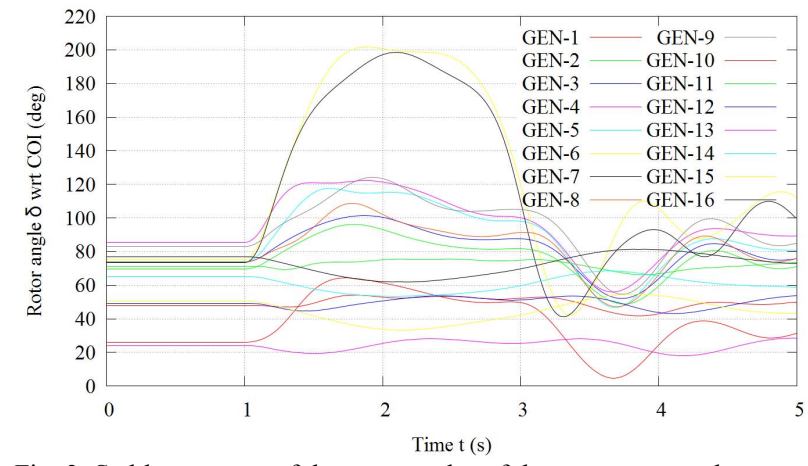

Fig. 3: Stable response of the rotor angles of the generators to the transient disturbance
Unstable case. In order to obtain an unstable case, the power flow of the marginal stable case was modified. To further stress the system, the power generation of the critical generators was increased. Therefore, the power generation of generator 4 was decreased by $10 \mathrm{MW}$ and at the same time the power output of generator 6 was increased by the same amount. This accounts to a change in power generation of approx. $1.5 \%$ at both generators. This modification of the power flow and the same fault as in the stable case triggered a transient first swing instability and the loss of synchronism of generators 6 and 7. The reference unstable case using $6^{\text {th }}$-order synchronous machine models and the rotor angles over time of all 16 generators can be seen in Figure 4.

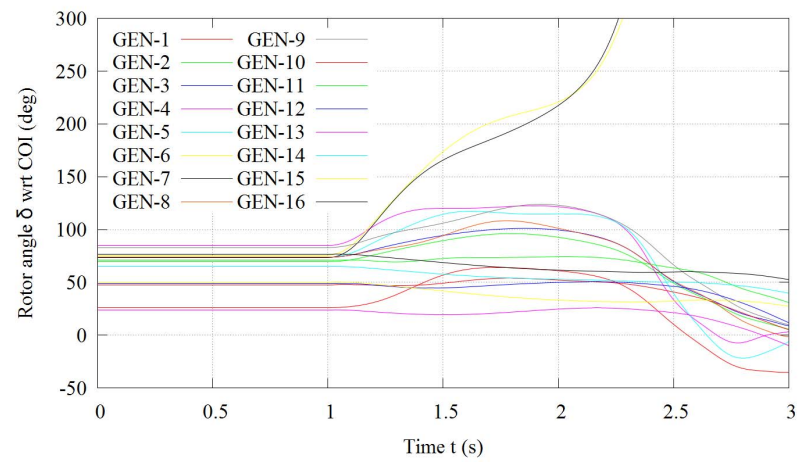

Fig. 4: Unstable response of the rotor angles of the generators to the transient disturbance

\section{Simulation results}

In the following section, the simulation results using the various generator models are discussed with respect to representation of the stability mechanism and possibility to assess transient stability using preventive SIME.

\section{Representation of the stability mechanism}

In order to detect instability correctly, the stability mechanism has to be depicted accurately. For the case of transient stability, the rotor angles of the machines allow to detect loss of synchronism and the origin of the stability problem. Hence, a comparison of the rotor angles may give an indication on the needed model detail. In the following, the rotor angle responses of the critical machines are compared when using varying SM models.

WSCC unstable and stable case. In the two cases generator 2 was identified as the critical machine. A comparison of the unstable rotor angle responses of generator 2 using the four different machine models is shown in Figure 5.

It can be seen that approximately for the first $400 \mathrm{~ms}$ after the fault, the development of the rotor angles of generator 2 over time are differing only slightly. However, thereafter the development begins to diverge. The generator appears to be first swing stable, when using low order generator models such as the $2^{\text {nd }}$ - and $3^{\text {rd }}$-order 
model. The simulation using a $4^{\text {th }}$-order model shows the same instability mechanism as the reference case using the $6^{\text {th }}$-order model. It even seems to be slightly pessimistic since the rotor angle is increasing faster than in the reference case.

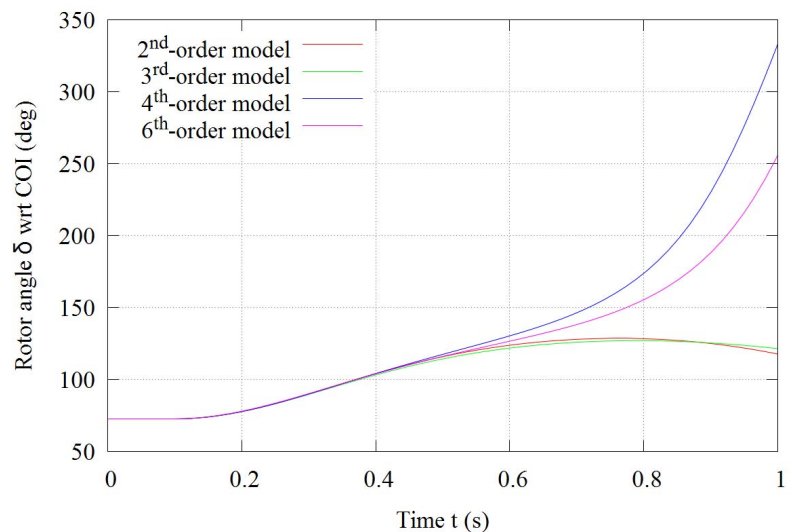

Fig. 5: Unstable case: Rotor angle response of generator 2 using the four different generator model details

A comparison of the rotor angle trajectories of the stable case lead to a similar conclusion. Figure 6 shows that the rotor angle trajectories are similar for the first $200 \mathrm{~ms}$ after the disturbance, but begin to deviate subsequently. The deviations for $2^{\text {nd }}$ - and $3^{\text {rd }}$-order models are larger than for the $4^{\text {th }}$-order model. However, the first swing characteristic is the same for all four models, where the $4^{\text {th }}$-order model is, with respect to the maximum angle excursion, slightly more pessimistic and the $3^{\text {rd }}$-order model slightly more optimistic.

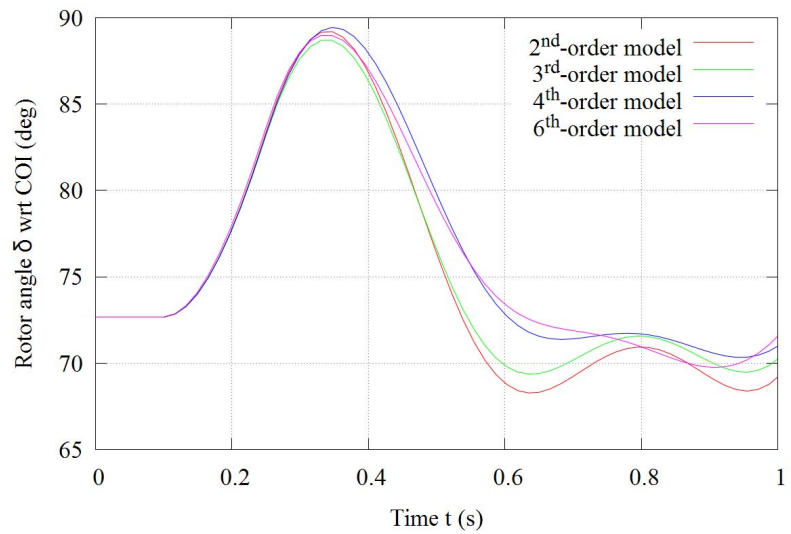

Fig. 6: Stable case: Rotor angle response of generator 2 using the four different generator model details

New England and New York. For the two scenarios of the second test system the generators 6 and 7 were identified as critical. Subsequently, the rotor angle trajectories of one of the critical generators (generator 6) are compared for the stable and unstable case. The different rotor angle curves are obtained from varying the SM model.

Figure 7 shows the rotor angle responses for the stable case and it can be seen that only the simulation using the second order model fails to represent the correct stability mechanism; meaning that all but the $2^{\text {nd }}$-order model simulation show a stable rotor response.
Approximately, for the first $400 \mathrm{~ms}$ the different rotor angle trajectories only vary slightly. However, afterwards a clear separation is visible. When excluding the $2^{\text {nd }}$-order model, it can be observed that with decreasing order of detail, the maximum return angle decreases and it seems that the angle response is becoming less critical.

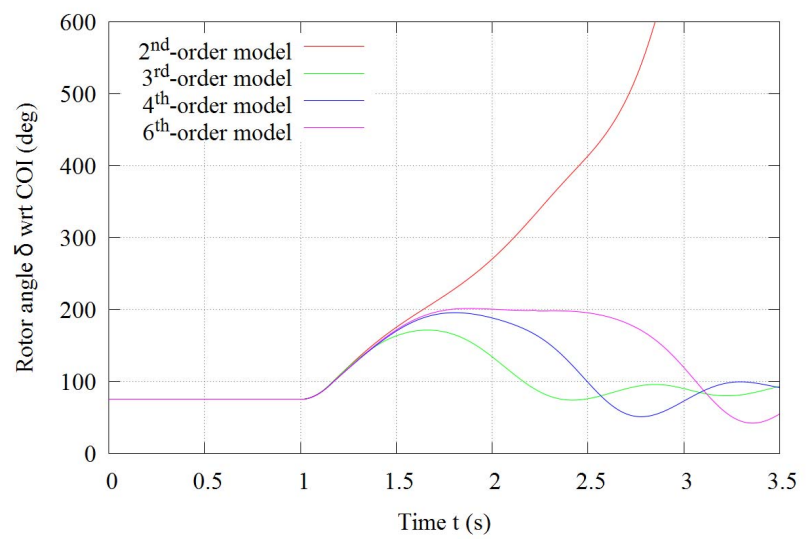

Fig. 7: Stable case: Rotor angle response of generator 6 using the four different generator models

Figure 8 shows the rotor angle response of generator 6 for the unstable case and varying model. Again it can be observed that for the first $400 \mathrm{~ms}$ the rotor angle trajectories vary only slightly. In this case only the simulation with $3^{\text {rd }}$-order model does not depict the correct instability mechanism, since the machine appears to be first swing stable. However, the simulations utilizing $2^{\text {nd }}$ - and $4^{\text {th }}$-order models show a similar behavior as the reference case with $6^{\text {th }}$-order model, namely the loss of synchronism of the generator within the first swing.

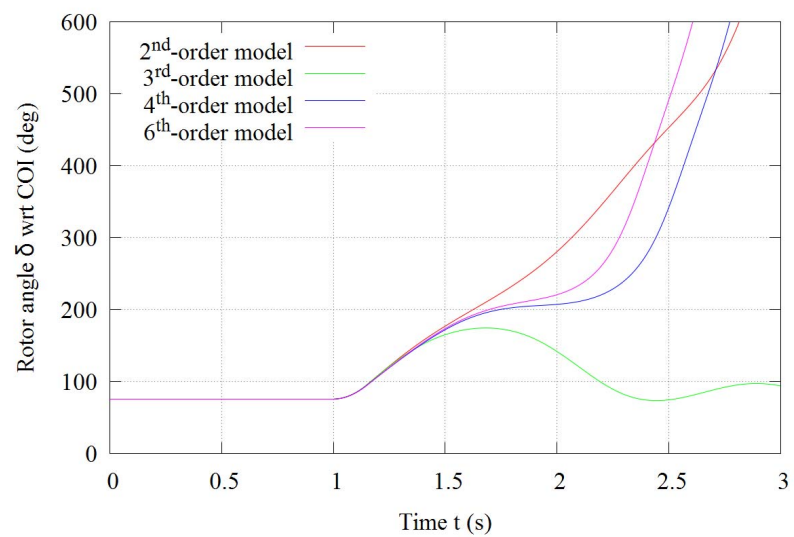

Fig. 8: Unstable case: Rotor angle response of generator 6 using the four different generator model details

\section{Stability Assessment Results using SIME}

Determination of the stability margin. In the following, the determined stability margins are analyzed for the discussed transient stability scenarios of the two test systems. The aim is to investigate if a reduced order model of a synchronous machine is sufficient to represent its dynamics during and following a transient disturbance. In the stable as well as in the unstable case of the WSCC test system, the estimation of the $P-\delta$ curve and the 
calculation of the stability margin led to the conclusion that only generator 2 is critical and, hence, the following analysis solely considers the stability assessment of this generator as the critical generator group.

It is expected that the estimation of the stability margin is improving with increasing number of considered simulated data. Furthermore, it is expected that the stability margin converges to a constant value. Figure 9 shows the determined margin for the unstable case. For the four simulations with differing generator model, it can be seen that the margins converge to a constant value.

The simulations, when using low order generator models, showed that the system is first swing stable in the unstable reference case (see Figure 5). The transient stability assessment of the first swing using SIME confirms this, since the stability margin converges to a positive value. The rotor angles over time for simulations with higher order generator model showed a loss of synchronism within the first swing. The TSA of these cases correctly predicts this loss of synchronism, which is apparent due to the convergence to a negative stability margin

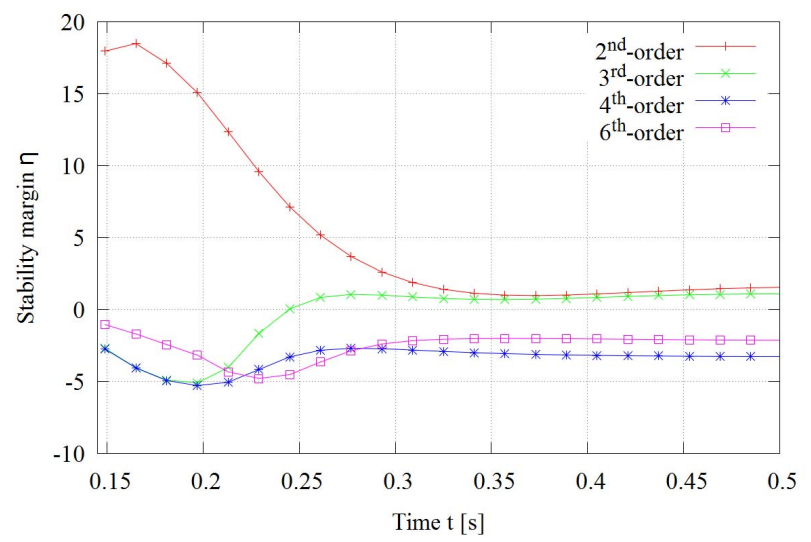

Fig. 9: Stability margins over time for the critical generator group consisting only of generator 2 and with differing degree of model detail

Consequently, SIME determined stability in the four simulations accurately. However, the stability mechanism is only depicted correctly with models of $4^{\text {th }}$-order and higher.

In the section analyzing the stability mechanism of the stable case, it was shown that the first swing characteristic was similar for all the generator models. Consequently, it is expected that SIME will determine all the four simulations to be first swing stable. Figure 10 shows the calculated stability margin of the stable case. Due to the rather fast first swing and the few data sets available to extrapolate the $P-\delta$ curve, the estimated stability margin seems to converge to a constant value, but does not reach it. Hence, for the presented stable case any of the considered synchronous machine models seems to be sufficient to assess stability.

For the case of the New England and New York system generator 6 and 7 were identified as the critical generator group in both scenarios. Since the stable case is marginally stable and the unstable case marginally unstable, it is expected that the stability margin will converge to a value very close to zero in both cases.
Figure 11 shows the computed stability margin over time of the stable case.

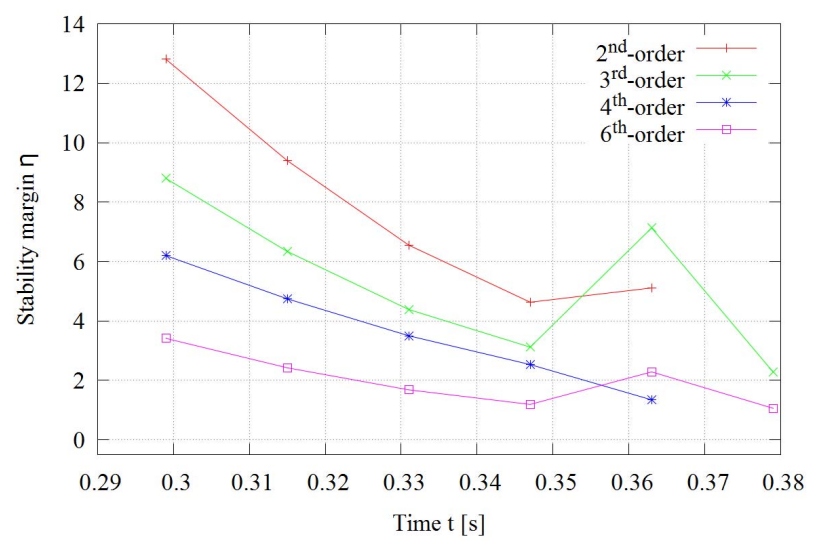

Fig. 10: Stability margins over time of the stable scenario for the critical generator group consisting only of generator 2 and with differing degree of model detail

The analysis of the rotor angle of generator 6 (see Figure 7) showed a stable response using SM models of $3^{\text {rd }}$-order and higher. Consequently, SIME determines those simulations as stable and only the simulation using a $2^{\text {nd }}$-order SM model as unstable.

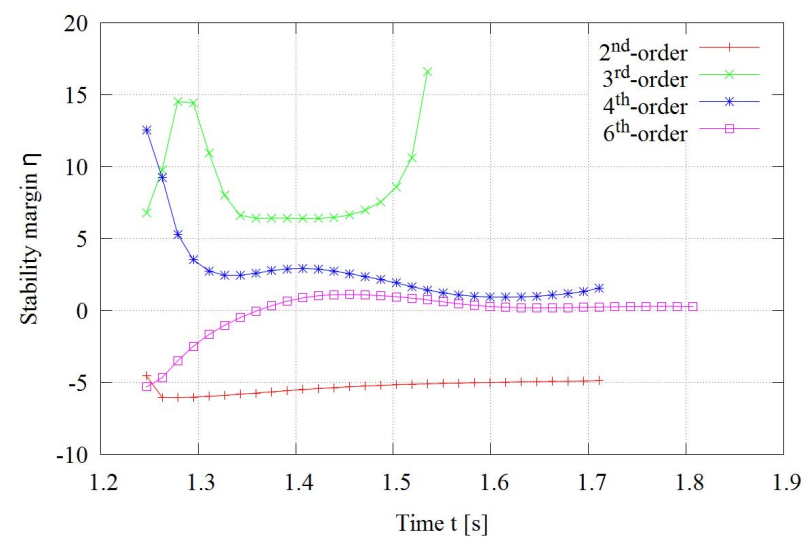

Fig. 11: Stability margins over time of the stable scenario for the critical generator group consisting of generator 6 and 7 with differing degree of model detail

The stability margin determined for the $2^{\text {nd }}$-order model simulation changes only slightly with time. This may be explained by the fact that TSAs using EAC are derived from the assumption of fixed voltage behind the transient reactance, which is also assumed for the case of a $2^{\text {nd }}$-order model. The stability margin of the simulation using a $3{ }^{\text {rd }}$-order model is the next fastest simulation to reach a (temporarily) constant stability margin. The subsequent rise of the stability margin may be explained by the observance that very close to the return angle the quadratic estimation of the $P_{a}-\delta$ curve is no longer sufficient. For the $4^{\text {th }}$-order model the stability margin remains positive until the end of the simulation. However, the stability margin does not reach a constant value. The stability margin computed from values of the simulation using $6^{\text {th }}$-order SM models begin with a negative margin, 
but it converges over time and with improving estimation of the $P_{a}-\delta$ curve to a slightly positive value.

Figure 12 shows the stability margin of the unstable case of the New England and New York system. Recall Figure 8 for this case all simulations but the simulation using $3{ }^{\text {rd }}$-order generator model depicted an unstable rotor angle response similar to the reference case. The stability margin of the $2^{\text {nd }}$-order simulation is slightly more negative than in the prior case, but as steady as before. The simulation with $3^{\text {rd }}$-order models is much too optimistic and the stability assessment indicates a stable case. With a fourth order model the stability margin begins with a positive value, but slowly converges to a slightly negative margin indicating an unstable case. The stability margin determined from the $6^{\text {th }}$-order model simulation begins with a negative margin and converges to a negative margin close to zero, which was expected since the case is marginal unstable. In this case the simulations with $4^{\text {th }}$ - and $6^{\text {th }}$-order models allowed a correct stability assessment, where the results with $4^{\text {th }}$-order were slightly more optimistic.

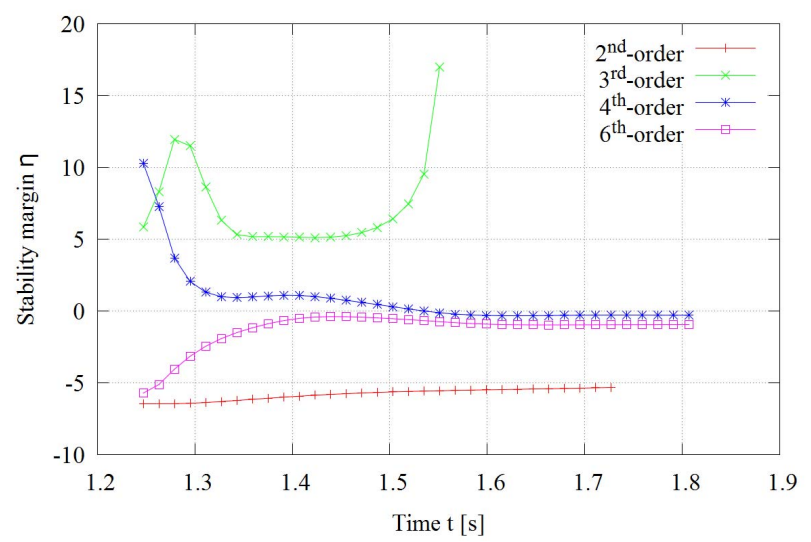

Fig. 12: Stability margins over time of the unstable case for the critical generator group consisting only of generator 6 and 7 with differing degree of model detail

\section{Conclusion}

The paper begins with a short description of the SIngle Machine Equivalent (SIME) method, which is based on the equal area criterion, and can be used to assess transient stability. SIME is a hybrid method combining the advantages of using time-domain simulation and using a direct method for transient stability assessment (TSA). In this paper SIME is used to carry out a fast transient stability screening of the current power system condition. For that purpose the computational burden of the TSA method should be minimized. In order to speed up the necessary time-domain simulation, a reduction of the synchronous machine model is considered in this work. The modeling degree of the synchronous generators was varied in two test power systems and two transient stability scenarios. For that purpose $6^{\text {th }}-, 4^{\text {th }}-, 3^{\text {rd }}-$ and $2^{\text {nd }}$-order models were considered, the stability mechanism by means of the rotor angle trajectories of the critical machines were compared and the resulting transient stability assessment results using the preventive
SIME implementation were investigated. In all cases the simulation with $6^{\text {th }}$-order model was used as reference.

The results showed that simulations using a synchronous machine model of $3^{\text {rd }}$-order and below do not exhibit the correct stability mechanism and, consequently, the transient stability assessment with SIME fails. Furthermore, it was shown that a representation of the synchronous machines by a $4^{\text {th }}$-order model may be sufficient, since the simulation displayed in all four cases the right stability mechanism and allowed to correctly determine transient stability. It should be mentioned that in the case of the New England and New York system the TSA with the $4^{\text {th }}$-order model led to slightly more optimistic stability assessment results than in the reference case. It should be noted the stable and unstable scenario of the New England and New York system were marginal stable and unstable and nevertheless, the $4^{\text {th }}$-order generator model representation was sufficient.

The simulation results at hand may suggest that a representation of the synchronous machine by their $4^{\text {th }}$-order model is sufficient to assess transient first swing stability.

\section{References}

[1] The Danish Ministry of Climate and Energy, "Energy Strategy 2050 - from coal, oil and gas to green energy," The Danish Ministry of Climate and Energy, Stormgade 2-6 1470 Copenhagen K Denmark, Tech. Rep., Feb. 2011.

[2] A. Phadke, J. Thorp, and M. Adamiak, "A New Measurement Technique for Tracking Voltage Phasors, Local System Frequency, and Rate of Change of Frequency," IEEE Trans. on Power App. and Syst., vol. 102, no. 5, pp. 1025-1038, May 1983.

[3] A. Phadke and J. Thorp, Synchronized Phasor Measurements and Their Applications. Springer Verlag, 2008.

[4] A. Phadke and R. De Moraes, "The Wide World of Wide-Area Measurement," IEEE Power and Energy Mag., vol. 6, no. 5, 2008.

[5] S. Skok, I. Ivankovic, and Z. Cerina, "Applications Based on PMU Technology for Improved Power System Utilization," in PES General Meeting, 2007. IEEE, Jun. 2007, pp. 1-8.

[6] D. Novosel, V. Madani, B. Bhargava, K. Vu, and J. Cole, "Dawn of the grid synchronization," IEEE Power and Energy Mag., vol. 6 , no. 1, pp. 49-60, Jan. 2008.

[7] H. Jóhannsson, "Development of early warning methods for electric power systems," Ph.D. dissertation, 2011.

[8] H. Jóhannsson, R. Garcia-Valle, T. Weckesser, A. Nielsen, and J. Østergaard, "Real-time stability assessment based on synchrophasors," in Proc. of 2011 IEEE Trondheim PowerTech, Jun. 2011, pp. 1-8.

[9] M. Glavic and T. Van Cutsem, "Wide-Area Detection of Voltage Instability From Synchronized Phasor Measurements. Part I: Principle," IEEE Trans. Power Syst., vol. 24, no. 3, pp. 14081416, Aug. 2009.

[10] —, "Wide-Area Detection of Voltage Instability from Synchronized Phasor Measurements. Part II: Simulation Results," IEEE Trans. Power Syst., vol. 24, no. 3, pp. 1417-1425, 2009.

[11] T. Weckesser, H. Jóhannsson, S. Sommer, and J. Østergaard, "Investigation of the Adaptability of Transient Stability Assessment Methods to Real-Time Operation," in IEEE Proc., Innovative Smart Grid Technologies, Oct. 2012, pp. 1-9.

[12] Fabozzi, D., Haut, B., Chieh, A. S., \& Van Cutsem, T. (2013). Accelerated and Localized Newton Schemes for Faster Dynamic Simulation of Large Power Systems. IEEE Transactions on Power Systems, 1-12.

[13] Kundur, P. Power System Stability and Control. (N. J. Balu \& M. G. Lauby, Eds.). McGraw-Hill Inc. 1994. 
[14] Athay, T., Podmore, R., \& Virmani, S. (1979). A practical Method for the Direct Analysis of Transient Stability. IEEE Transactions on Power Apparatus and Systems, PAS-98(2), 573-584.

[15] M. Pavella, D. Ernst, and D. Ruiz-Vega, Transient Stability of Power Systems: A Unified Approach to Assessment and Control. Kluwer Academic Publishers, 2000.

[16] Chiang, H.-D., Wu, F. F., \& Varaiya, P. P. (1994). A BCU Method for Direct Analysis of Power System Transient Stability. IEEE Transactions on Power System, 9(3), 1194-1208.

[17] Xue, Y., \& Pavella, M. (1989). Extended equal-area criterion: an analytical ultra-fast method for transient stability assessment and preventive control of power systems. International Journal of Electrical Power \& Energy Systems, 11(2), 131-149.

[18] Glavic, M., Ernst, D., Ruiz-Vega, D., Wehenkel, L., \& Pavella, M. (2007). E-SIME - A Method for Transient Stability Closed-Loop Emergency Control : Achievements and Prospects Fundamentals of E-SIME. 2007 iREP Symposium- Bulk Power System Dynamics and Control - VII, Revitalizing Operational Reliability.

[19] P. Sauer and M. A. Pai, Power System Dynamics and Stability, Prentice Hall, 1998.

[20] IEEE Std 1110-2002 (Revision of IEEE Std 1110-1991). (2003). IEEE Guide for Synchronous Generator Modeling Practices and Applications in Power System Stability Analyses. doi:10.1109/IEEESTD.2003.94408

[21] Rogers, G. (2000). Power System Oscillations (p. 328). Springer. 